\title{
New technical trends in cancer research: the laser microdissection approach
}

\author{
Keywords: quality of life, treatment, spiritual, worsens, \\ molecular, biology, heterogeneity
}

\author{
Abbreviations: PET, positron emission tomography; \\ ${ }^{18} \mathrm{FDG},{ }^{18} \mathrm{f}$-labelled-2-fluoro-2-deoxy-D-Glucose; LOH, loss of \\ heterozygosity; IHC, immunohistochemistry
}

\section{Mini review}

Palliative care is attention given to improve the quality of life of patients who have a serious or life-threatening disease, such as cancer. The goal of palliative care is to prevent or treat, as early as possible, the symptoms and side effects of the disease and its treatment, in addition to the related psychological, social, and spiritual problems. The main aim of medicine, usually called personalized medicine, and medical research is precisely to prevent people from falling into such a situation of stress, suffering and despair. It is therefore essential to detect as soon as possible the first symptoms of a disease that worsens if it is not considered at the earliest step and will precipitate these people into this "black corridor".

For almost twenty years now, huge advances in the field of molecular biology have made it possible to identify molecules or molecular markers that represent the signature of a serious pathology, I mean cancer in particular. Thus, rapid detection enables to envisage first-line treatments such as surgical ablation, for example, which would avoid treatment that is heavier and more invasive for the patient.

Mentioned for the first time there are more than thirty years, the concept of intratumoral heterogeneity experienced a revival since. ${ }^{1}$ The observation that within the same primary tumor coexist populations of cancer cells with different evolutionary path was not possible before the advent of the new generation of sequencing technologies. Spatial heterogeneity of tumors has been identified within and between metastatic lesions and can be visualized with targeted PET imaging, ${ }^{2}$ immunohistochemistry (or IHC, is a technique used to determine the presence and level of specific cellular proteins. IHC measures protein expression using specially labeled antibodies that can bind to the proteins of interest) and micro dissected-targeted NGS. ${ }^{3}$

In this short review we envisage the approach of the disease and its evolution by the technique of laser-assisted micro dissection. Laser micro dissection technologies, involving direct viewing and expertise by a pathologist, enable access to defined cell populations or specific region on any type of tissue sample, thus selecting nearpure populations of targeted cells. It opens the way for molecular methods directed towards well-defined populations, and provides also a powerful tool in studies focused on a limited number of cells. Laser micro dissection has wide applications in oncology (diagnosis and research) with applied cellular and molecular biology, and biochemistry for tissue selection. Other areas have been gradually opened up to these new methodological approaches, such as cell cultures $^{4}$ and cytogenetics. ${ }^{5}$ In clinical oncology trials, molecular profiling of micro dissected samples can yield global "omics"
Volume I Issue 3 - 2017

\author{
Luc G Legrès \\ Laboratory of Pathology, Institute of Hematology, France
}

Correspondence: Luc G Legrès, Laboratory of Pathology, Institute of Hematology, Inserm UMR S I I 65 / University of Paris-Diderot, Saint-Louis Hospital, Paris, France, Email luc.legres@inserm.fr

Received: May 19, 2017| Published: August 03, 2017

information (genomic, transcriptomic and proteomic mainly) which, together, with the morphological analysis of cells, can provide the basis for diagnosis, prognosis and patient-tailored treatments.

Cells isolated by micro dissection have been widely used as a genomic DNA isolation tool for library generation. In molecular biology, a DNA library is a collection of DNA fragments of one organism, each usually carried by a plasmid (an extra chromosomal self-replicating structure of bacterial cells that carries genes for a variety of functions not essential for cell growth and that can be transferred to other cells by conjugation or transduction) that is stored and propagated in a population of micro-organisms (bacteria or yeast) through the process of molecular cloning. As the population of microorganisms is grown in culture, the DNA molecules contained within them are copied and propagated (thus, "cloned"). A DNA probe, as DNA sequence labelled with a radioactive element, is used then to locate a specific DNA sequence in the library by binding to it.

Although a large number of studies have described analyses of DNA and RNA extracted from laser-captured tissue fragments, protein or lipid-based studies are still scarce. In addition, biopsies performed nowadays are often radiologically guided needle biopsies yielding smaller amounts of tissue than former surgical procedures. ${ }^{6}$ Functional imaging is a new tool that improves the diagnostic return of biopsies and assists the radiologist in choosing the best target. The Positron Emission Tomography (PET) imaging is a noninvasive diagnostic tool that provides tomographic images and can be used to obtain quantitative parameters concerning the metabolic activity of target tissues. The most commonly used tracer at present is the ${ }^{18} \mathrm{~F}$-labelled-2-fluoro-2-deoxy-D-glucose $\left({ }^{18} \mathrm{FDG}\right) .{ }^{7} \mathrm{FDG}$ is an analogue of glucose and is taken up by living cells via cell membrane glucose transporters and subsequently incorporated into the first step of the normal glycolytic pathway. 8 Increased tumoral uptake of ${ }^{18} \mathrm{FDG}$ reflects elevated glucose consumption by tumor cells, as evidenced by the overexpression of glucose transporter proteins at the cells surface and increased levels of active hexokinase demonstrated in many tumors. ${ }^{9}$ In the understanding of cancer, the difference between PET and the various conventional radiologic imaging techniques, such as computed tomography or conventional magnetic resonance imaging, is that the former rates metabolic characteristics of the tumour, whereas the latter predominantly review the tumor's anatomical or morphologic structures. In many circumstances, this 
characteristic permits a more accurate evaluation after treatment and enables early detection of cancerous lesions. Anatomical and morphological information derived from CT can be used to improve the localization, and together ${ }^{18} \mathrm{FDG}-\mathrm{PET} / \mathrm{CT}$ can be used to select the lesions that were hyper metabolic or parts of tumors that take up most ${ }^{18} \mathrm{FDG}$. Biopsies could therefore be orientated towards this area. This powerful tool has reduced the amount of material collected while improving its quality, enabling the performance of more sophisticated and reproducible analyses with few post-operative complications for the patient. Image-guided biopsy is a powerful tool but it is necessary to highlight the importance of a coordinated approach between radiologists, pathologists and referring clinicians to optimize the diagnosis of diseases.

Tumoral tissues often contain a wide array of tumor cells disseminated in an abundant connective tissue called the stroma. Stromal tissue is composed of fibrous tissue, vessels and inflammatory cells and possibly necrotic or haemorrhagic areas. Laser micro dissection overcomes the problem of cellular heterogeneity that also characterizes tumoral tissues. It aims to recover a target cluster of cells, or a single cell precisely selected under microscope guidance, from a complex frozen or fixed by prior paraffin-embedded tissue sections for subsequent molecular analysis. ${ }^{10,11}$ Biological samples often need to be solidified to allow fine sectioning for light microscopy observations. Paraffin wax is the most frequently used hard matrix for cutting after fixation by formalin or formalin derivatives. However, formalin, like other aldehyde fixatives, forms cross-linked methylene bridges and Schiff bases between basic amino acid (lysine) residues of proteins. ${ }^{12}$ The changes in immunoreactivity observed after
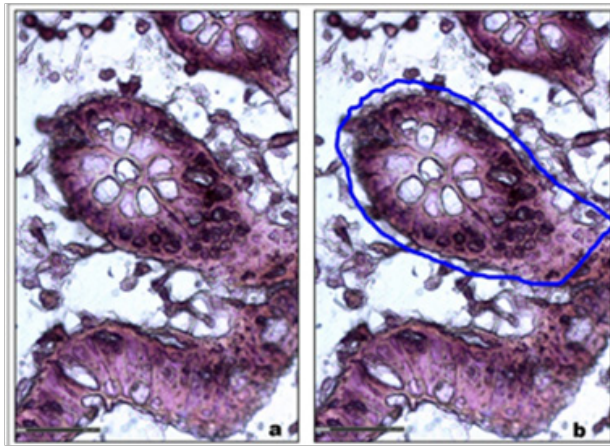

Figure I A.Tracking of the region of interest.

B. Graphic delineation of the area of interest (blue line).

C. Fine and precise cutting laser made of the area.

D. Recovery of the area of interest for molecular analyzes.

Insert: Microdissected sample into the cap of a tube.

Neoplasia is the main field of application of this technique for selecting tissue. One can analyze the expression of many genes in a single reaction quickly and in an efficient manner. From cell fragments, one of the most common applications has been the search for loss of heterozygosity $(\mathrm{LOH})$ in malignant tumors. This type of study is based on individual DNA polymorphisms. Thus, tissue micro dissection has been widely used in lung, ${ }^{17}$ colon $^{18}$ or prostate cancer. ${ }^{19}$ In our laboratory, we have shown that tissue micro dissection greatly increased the sensitivity of $\mathrm{LOH}$ detection in inflammatory breast cancer. ${ }^{20}$ Allelic loss detection with laser micro dissection in breast cancer were corroborated by Dias MF et al. ${ }^{21}$ Their data indicate that formaldehyde fixation are explained by a reduced availability for the secondary antibody which is masked by cross-linking of globular proteins. ${ }^{13}$ Thus, snap-freezing in liquid nitrogen, a process that brings a sample to very low temperature at $-196^{\circ} \mathrm{C}$ is favored to preserve the structure of the sample (e.g. RNA, protein, or live cells). For both kinds of sample, fixed or freezed, thin slices improve the access of dyes, probes, and antibodies for DNA, RNA or proteins detections. Developed in 1996, this technique for isolating specific cells from a sample responds to the need for miniaturization of analytical techniques applicable to very small cell numbers. ${ }^{14}$

The intratumoral heterogeneity is a familiar concept to the pathologist. Under the microscope, the cancerous cells within the same tumor are often characterized by a morphological variability and diversity of expression of molecular markers..$^{15}$ Medical practices are disrupted by the advent of therapies targeting specific molecular defects in each tumor type. The impact of these data on the mechanisms governing the formation of a tumor and its metastasis is thus of crucial importance for our understanding of the disease.

For patient diagnosis and experimental studies, biological tissue can be either analyzed under a microscope after immuno-histostaining or crushed for further molecular analysis. Laser micro dissection provides a valuable link between these two approaches. It gives new insights into cellular mechanisms, genetic disorders, tumor biomarker identification patient-tailored therapy ${ }^{16}$ Tissue micro dissection is used to obtain phenotypically identical cells, while other cells can be ignored or destroyed, therefore increasing the sensitivity and specificity of subsequent molecular analyses (Figure 1).

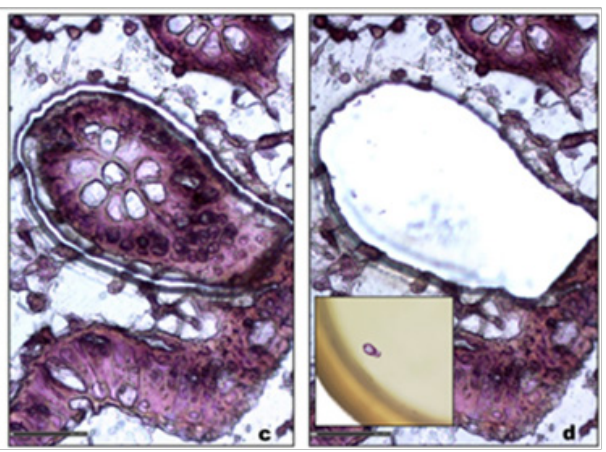

the intratumoral heterogeneity occurs very early in the progression of cancer associated with chromosomal alterations showing that different clones of cells co-exist within a single tumor. DNA Microarray technology has empowered the scientific community to understand the fundamental aspects underlining the growth and development of life as well as to explore the genetic causes of anomalies. Now, with the evolution of microarray technology, it will be possible for the researchers to further classify the types of cancer on the basis of the patterns of gene activity in the tumor cells. Combination of micro dissection and microarray analysis can for example enable comparison of gene expression in different breast cancer areas, such as the intra- 
ductal component or the infiltrating component, with greater accuracy than global gene expression analyses, ${ }^{22}$ as it is also demonstrated in different cell populations from the same sample, such as tumor cells and normal cells in colorectal cancer. ${ }^{23}$

The technique of laser micro dissection is of great importance in the field of molecular analysis at the level of the cell. It helped to bring greater precision in the selection of biological tissues that can be seen under a microscope according to their physiological or pathological state or not. This precision in the recovery of biological samples is primarily a function of expertise in the recognition of biological tissues. The ability to use different markers as specific dyes or not, conjugated-antibody or not, has extended greatly the scope for future research from frozen or even paraffin-embedded tissue after fixation. Then, the very fine cutting laser beam and the ability to avoid or destroy any unwanted cells or cell areas only strengthen the precision, quality and reproducibility of the analyzes made on these samples later. The laser-capture micro dissection process does not alter or damage the morphology or chemistry of the sample collected, nor the surrounding cells. This represents a major step forward in many areas of application to obtain better results from very small sample. Taken together, the technique of laser-assisted tissue micro dissection has obvious advantages in the molecular analysis of very small samples corroborated by numerous publications showing high rates of accuracy. On a general basis, the development of an optimal protocol for any tissue procurement is therefore a prerequisite, providing guidelines for clinicians and researchers for satisfactory preparation of tissues and an optimization of the classic techniques for molecular analysis. These analyzes can be used clinically as aids in cancer diagnosis, clinical management, genomic profile studies, and targeted therapy.

The identification of genomic abnormalities, genetic or epigenetic tumors can now establish prognosis for individual patients and predict susceptibility and resistance to many anticancer drugs, including new targeted therapies. In the near future, increasing numbers of research laboratories will develop high-level platforms for such studies on patients entering clinical trials. Ultimately, validation of this technique will enable individualized therapeutic approaches and thus better management of patients.

\section{Acknowledgements}

None.

\section{Conflict of interest}

The author declares no conflict of interest.

\section{References}

1. Gerlinger M, Rowan AJ, Horswell S, et al. Intratumor heterogeneity and branched evolution revealed by multiregion sequencing. $N$ Engl J Med. 2012;366(10):883-892.

2. Wong ANM, McArthur GA, Hofman MS, et al. The advantages and challenges of using FDG PET/CT for response assessment in melanoma in the era of targeted agents and immunotherapy. Eur J Nucl Med Mol Imaging. 2017;44(Suppl 1):67-77.

3. Rosenberg AZ, Armani MD, Fetsch PA, et al. High-throughput microdissection for next-generation sequencing. PloS One. 2016;11(3):e0151775.

4. Vogel A, Horneffer V, Lorenz K, et al. Principles of laser microdissection and catapulting of histologic specimens and live cells. Methods Cell Biol. 2007;82:153-205.
5. Meltzer PS, Guan XY, Burgess A, et al. Rapid generation of region specific probes by chromosome microdissection and their application. Nat Genet. 1992;1(1):24-28.

6. de Kerviler E, Benet C, Brière J, et al. Image-guided needle biopsy for diagnosis and molecular biology in lymphomas. Best Pract Res Clin Haematol. 2012;25(1):29-39.

7. Juweid ME, Cheson BD. Positron-emission tomography and assessment of cancer therapy. N Engl J Med. 2006;354(5):496-507.

8. Som P, Atkins HL, Bandoypadhyay D, et al. A fluorinated glucose analog, 2-fluoro-2-deoxy-D-glucose (F-18): nontoxic tracer for rapid tumor detection. J Nucl Med Off Publ Soc Nucl Med. 1990;21(7):670-675.

9. Bos R, van Der Hoeven JJM, van Der Wall E, et al. Biologic correlates of (18)fluorodeoxyglucose uptake in human breast cancer measured by positron emission tomography. J Clin Oncol Off J Am Soc Clin Oncol. 2012;20(2):379-387.

10. Bertheau P, Meignin V, Janin A. Microdissection on histologic and cytologic preparation: an approach to tissue heterogeneity. Ann Pathol. 1998;18(2):110-119.

11. Simone NL, Bonner RF, Gillespie JW, et al. Laser-capture microdissection: opening the microscopic frontier to molecular analysis. Trends Genet TIG. 1998;14(7):272-276.

12. Metz B, Kersten GFA, Hoogerhout P, et al. Identification of formaldehydeinduced modifications in proteins: reactions with model peptides. $J$ Biol Chem. 2004;279(8):6235-6243.

13. Mason JT, O’Leary TJ. Effects of formaldehyde fixation on protein secondary structure: a calorimetric and infrared spectroscopic investigation. $J$ Histochem Cytochem Off $J$ Histochem Soc. 1991;39(2):225-229.

14. Emmert-Buck MR, Bonner RF, Smith PD, et al. Laser capture microdissection. Science. 1996;274(5289):998-1001.

15. Marusyk A, Almendro V, Polyak K. Intra-tumour heterogeneity: a looking glass for cancer. Nat Rev Cancer. 2012;12(5):323-334.

16. Domazet B, Maclennan GT, Lopez-Beltran A, et al. Laser capture microdissection in the genomic and proteomic era: targeting the genetic basis of cancer. Int J Clin Exp Pathol. 2008;1(6):475-488.

17. Mead LJ, Gillespie MT, Hung JY, et al. Frequent loss of heterozygosity in early non-small cell lung cancers at chromosome $9 \mathrm{p} 21$ proximal to the CDKN2a gene. Int J Cancer. 1997;71(2):213-217.

18. Zhuang Z, Emmert-Buck MR, Roth MJ, et al. von Hippel-Lindau disease gene deletion detected in microdissected sporadic human colon carcinoma specimens. Hum Pathol. 1996;27(2):152-156.

19. Vocke CD, Pozzatti RO, Bostwick DG, et al. Analysis of 99 microdissected prostate carcinomas reveals a high frequency of allelic loss on chromosome 8p12-21. Cancer Res. 1996;56(10):2411-2416.

20. Bertheau P, Plassa LF, Lerebours F, et al. Allelic loss detection in inflammatory breast cancer: improvement with laser microdissection. Lab Invest. 2001;81(10):1397-1402.

21. Dias MF, Blumenstein R, Russo J. Use of laser capture microdissection allows detection of loss of heterozygosity in chromosome $9 \mathrm{p}$ in breast cancer. Oncol Lett. 2017;13(5):3831-3836.

22. Zhu G, Reynolds L, Crnogorac-Jurcevic T, et al. Combination of microdissection and microarray analysis to identify gene expression changes between differentially located tumour cells in breast cancer. Oncogene. 2003;22:3742-3748.

23. Berdiel-Acer M, Cuadras D, Díaz-Maroto NG, et al. A monotonic and prognostic genomic signature from fibroblasts for colorectal cancer initiation, progression, and metastasis. Mol Cancer Res. 2014;12(9):1254-1266. 